\title{
Effects of Antioxidant Vitamins, Curry Consumption, and Heavy Metal Levels on Metabolic Syndrome with Comorbidities: A Korean Community-Based Cross-Sectional Study
}

\author{
Hai Nguyen Duc (D), Hojin Oh and Min-Sun Kim *(D)
}

check for updates

Citation: Duc, H.N.; Oh, H.; Kim, M.-S. Effects of Antioxidant Vitamins, Curry Consumption, and Heavy Metal Levels on Metabolic Syndrome with Comorbidities: A Korean Community-Based Cross-Sectional Study. Antioxidants 2021, 10, 808. https: / / doi.org/10.3390/ antiox10050808

Academic Editors: Silvia M. Arribas and Maria Angeles Martín-Cabrejas

Received: 3 April 2021

Accepted: 18 May 2021

Published: 19 May 2021

Publisher's Note: MDPI stays neutral with regard to jurisdictional claims in published maps and institutional affiliations.

Copyright: (c) 2021 by the authors. Licensee MDPI, Basel, Switzerland. This article is an open access article distributed under the terms and conditions of the Creative Commons Attribution (CC BY) license (https:// creativecommons.org/licenses/by/ $4.0 /)$.
Department of Pharmacy, College of Pharmacy and Research Institute of Life and Pharmaceutical Sciences, Sunchon National University, Sunchon 57922, Korea; haiyds@gmail.com (H.N.D.); navytoto@hanmail.net (H.O.) * Correspondence: minsun@scnu.ac.kr; Tel.: +82-102-510-1635

\begin{abstract}
The burden of metabolic syndrome (MetS) has increased worldwide, especially during the COVID-19 pandemic, and this phenomenon is related to environmental, dietary, and lifestyle risk factors. We aimed to determine the association between the levels of serum heavy metals, hs-CRP, vitamins, and curry intake and to predict risks of MetS based on marginal effects. A data set of 60,256 Koreans aged $\geq 15$ years between 2009 and 2017 was used to obtain information on sociodemographic, lifestyle, family history characteristics, MetS, food intake survey, and serum heavy metals. Daily intake of vitamins was measured by a one-day $24 \mathrm{~h}$ recall, and curry consumption was calculated using a food frequency questionnaire. Serum heavy metal levels were quantified by graphite furnace atomic absorption spectrometry and using a mercury analyzer. We found that vitamin B1, B2, B3, C, and A intakes were significantly lower in subjects with than without MetS. In contrast, serum levels of $\mathrm{Pb}, \mathrm{Hg}, \mathrm{Cd}$, vitamin $\mathrm{A}, \mathrm{E}$, and hs-CRP were significantly higher in subjects with MetS. The risk of MetS was significantly lower for high curry consumers than low curry consumers (adjusted odds ratio $0.85,95 \% \mathrm{CI} 0.74-0.98$ ). The risks of MetS were reduced by $12 \%$ and $1 \%$, when vitamin B1 and C intakes increased by one $\mathrm{mg}$, respectively, but were increased by $14 \%$, $3 \%$, and $9 \%$, when serum levels of $\mathrm{Pb}, \mathrm{Hg}$, and hs-CRP increased by one unit. These results show that the potential health benefits resulting from vitamin and curry intakes could protect the public against the dual burden of communicable and non-communicable diseases. Further studies are required to reduce risk factors associated with serum heavy metal levels and to determine whether interactions between vitamin and curry consumption influence the presence of MetS.
\end{abstract}

Keywords: antioxidant vitamins; heavy metals; curry consumption; metabolic syndrome; comorbidities

\section{Introduction}

Food is now understood to be a significant modifiable contributor to chronic disease, and empirical evidence indicates that dietary modifications may have positive or negative impacts on lifelong health [1]. Consumption of high-saturated-fat and high-energy diets, overuse of tobacco and alcohol, and sedentary lifestyles have contributed to increases in non-communicable diseases (NCDs), especially metabolic syndrome (MetS) [1,2]. MetS is a collection of metabolic disorders-that is, insulin resistance, dyslipidemia, central obesity, and hypertension - and a risk factor for the development of type 2 diabetes and cardiovascular diseases [3]. Of note, recent evidence suggests that MetS affects the progression and prognosis of COVID-19, and its severity has been correlated with poorer COVID-19 outcomes [4,5].

In addition to lifestyle and genetic factors, heavy metals are also risk factors of MetS [6-9]. Levels of heavy metals, especially lead $(\mathrm{Pb})$, mercury $(\mathrm{Hg})$, and cadmium (Cd), released into the environment by vehicles or factories or in contaminated seafood are 
increasing and accumulate in bones, kidney cortices, and lungs [10]. Heavy metals catalyze the release of reactive oxygen species (ROS), inflammatory mediators, and antithrombotic substances that damage vascular endothelial cells and exacerbate hypertension $[7,11]$. In particular, $\mathrm{Pb}$ and $\mathrm{Cd}$ disrupt blood clotting and increase the risk of CVD [7,12], while $\mathrm{Hg}$ accelerates carotid atherosclerosis [6,13].

Increasing evidence shows that vitamin intake could reverse CVDs, diabetes, and mental illness [14], and curry rice (the distinct yellow color of curry rice is mostly derived from a polyphenolic compound, curcumin [1,7-bis(4-hydroxy-3-methoxyphenyl)-1,6-heptadien3,5-dione]) has been shown to prevent and to be useful for treating CVDs, due to its antioxidant and anti-inflammatory properties [15]. In this study, we consider whether increased intake of vitamins and curry reduces the risk of MetS in the Korean population with or without various NCDs, and whether serum heavy metals and hs-CRP are positively associated with the risk of MetS.

\section{Materials and Methods}

\subsection{Study Population}

We used data obtained by KNHANES studies conducted by the Korean Ministry of Health and Welfare, specifically KNHANES IV (2009), KNHANES V (2010-2012), KNHANES VI (2013), and the KNHANES VII (2016-2017) [16]. These studies were conducted using a stratified, multi-stage, cluster-sampling method and involved consideration of geographic zone, level of urbanization, economic development, gender, and age distribution. Subjects surveyed were randomly selected from 10,533 households (2009), 8958 (2010), 8518 (2011), 8058 (2012), 8018 (2013), 8150 (2016), and 8127 (2017). In the present study, subjects that participated in a health interview and nutrition survey and underwent a health examination survey with adequate information on MetS were selected. Of the 60,362 individuals that participated in a KNHANES survey from 2009 to 2013 and 2016 to 2017, 106 were excluded for missing MetS information, and thus, 60,256 were eligible for data analysis. Written informed consent was required from patients and family members, and informed parental consent was obtained on behalf of all minors before examinations, which were performed by the Health and Nutrition Examination Department of the Korea Centers for Disease Control and Prevention (KCDC). A detailed description of the objectives and methods used and of the legal approvals obtained can be found on the KNHANES website (http: / / knhanes.cdc.go.kr/, accessed on 17 December 2020). This study was approved by the KNHANES inquiry commission and the Institutional Review Board (IRB) of Sunchon National University and followed the guidelines set out in the Declaration of Helsinki. From 2007 to 2013, KNHANES surveys were conducted with the approval of the IRB of the KCDC (2009-01CON-03-2C, 2010-02CON-21-C, 2011-02CON-06-C, 2012-01EXP-012C, 2013-07CON-03-4C, 2013-12EXP-03-5C). However, in 2016 and 2017, KNHANES was conducted without such approval in accord with the opinion of the IRB of the KCDC [17].

\subsection{Parameters}

Information on sociodemographic characteristics, lifestyle, current medications, medical, and family history was collected during health interviews. Alcohol intakes were classified as low or high (high-risk drinking was defined as $>5$ drinks per day for $\geq 1$ month). Subjects with a lifetime history of smoking of $>100$ cigarettes and who still smoked daily or occasionally were classified as current smokers; others were classified as ex-/non-smokers.

The physical activity was measured using the modified Global Physical Activity Questionnaire (GPAQ) developed by the WHO, which contains the groups of resistance exercise and weekly walking added to the original GPAQ. Physical activity was dichotomized as regular or irregular. Regular physical activity was defined as: (1) participation in vigorous, $\geq 20$ min per session for $\geq 3$ days a week; (2) or participation in moderate, $\geq 30$ min per session for $\geq 5$ days per week, (3) or participation in walking; $\geq 30$ min per session for $\geq 5$ days a week [18]. 
Dyslipidemia was defined as the presence of at least one of the following: LDL-C $\geq$ $160 \mathrm{mg} / \mathrm{dL}$, triglyceride $\geq 200 \mathrm{mg} / \mathrm{dL}$, HDL-C $<40 \mathrm{mg} / \mathrm{dL}$. Hypertension was defined as a systolic blood pressure (SBP) of $\geq 140 \mathrm{mmHg}$ or a diastolic blood pressure of $\geq 90 \mathrm{mmHg}$ or being on anti-hypertensive medication. Type 2 diabetes mellitus was defined as a fasting plasma glucose of $\geq 126 \mathrm{mg} / \mathrm{dL}$, receipt of anti-diabetic medication, or a $\mathrm{HbA} 1 \mathrm{c}$ of $\geq 6.5 \%$. Stroke, angina, myocardial infarction (MI), MI or angina, and other conditions were defined based on physicians' diagnoses and the presence of these conditions. Depression was also defined based on physicians' diagnoses, the presence or treatment for depression, or experience of depression during the previous year or of despair to the point that it disturbed daily routine for $\geq 2$ consecutive weeks [19]. Comorbidities were defined as any disease, such as CVDs, hypertension, hyperlipidemia, type 2 diabetes, cancers, thyroid, kidney, arthritis, osteoarthritis, rheumatoid arthritis, or depression, accompanying MetS. A family history of cardiovascular disease was defined as having at least one parent or sibling with a diagnosis of hypertension, ischemic heart disease, or stroke. A family history of type 2 diabetes or hyperlipidemia was defined as having at least one parent or sibling with a diagnosis of type 2 diabetes or hyperlipidemia.

\subsection{Laboratory Measurements}

Height, weight, waist circumference, and blood pressure were measured during medical checkups using standard procedures. BMI $\left(\mathrm{kg} / \mathrm{m}^{2}\right)$ was calculated by dividing weight $(\mathrm{kg})$ by height ${ }^{2}\left(\mathrm{~m}^{2}\right)$. Waist circumstance $(\mathrm{cm})$ was measured at the midpoint between the bottom of the rib cage and the iliac crest on the mid-axillary line when exhaling. Blood pressures were measured three times with 5-min intervals using a mercury sphygmomanometer with subjects seated after a 5 -min stabilization period. Blood pressures used in the analysis were the averages of second and third measurements. Blood samples were collected after $\geq$ an $8 \mathrm{~h}$ fast and analyzed at the Neodin Medical Institute in Korea. An enzymatic assay was used to determine total cholesterol, high-density lipoprotein cholesterol (HDL-C), triglyceride, low-density lipoprotein cholesterol (LDL-C), and fasting blood glucose levels using a Hitachi automated analyzer 7600 (Hitachi, Japan). The HbA1c level was measured using high-performance liquid chromatography (HLC-723G7; Tosoh, Tokyo, Japan). Serum highly sensitive C-reactive Protein (hs-CRP) was determined using Cobas (Cobas; Roche, Germany) and a Cardiac C-reactive Protein Highly Sensitive kit (Cardiac C-reactive Protein Highly Sensitive; Roche, Germany). The reference normal range for hs-CRP is 0 to $3 \mathrm{mg} / \mathrm{L}$ [20].

\subsection{Determination of Serum $\mathrm{Pb}, \mathrm{Hg}$, and $\mathrm{Cd}$ Levels}

$\mathrm{Pb}, \mathrm{Hg}$, and $\mathrm{Cd}$ analyses were performed as previously described [9]. In brief, these tests were performed by the Neodin Medical Institute, which had been approved by the Korean Ministry of Labor for Heavy Metal Analysis. Furthermore, the tests used met the requirements of the Korea Occupational Safety and Health Administration, the German External Quality Assessment Scheme, and the U.S. CDC. Pb and Cd levels were measured by graphite furnace atomic absorption spectrometry (model AAnalyst 600; Perkin Elmer, Turku, Finland) using Zeeman background correction, and total $\mathrm{Hg}$ levels were determined using a direct mercury analyzer (model DMA- 80 Analyzer; Bergamo, Italy) and the gold amalgam method (KCDC 2013). Limits of detection (LODs) were $0.223 \mu \mathrm{g} / \mathrm{dL}, 0.05 \mu \mathrm{g} / \mathrm{L}$, and $0.087 \mu \mathrm{g} / \mathrm{L}$ for $\mathrm{Pb}, \mathrm{Hg}$, and $\mathrm{Cd}$, respectively. Commercial standard reference materials purchased from Bio-Rad were used for internal quality assurance and control (Lyphocheck Whole Blood Metals Control; Bio-Rad, Hercules, CA, USA).

\subsection{Urinary Cotinine and Smoking Verification}

Spot urinary samples were collected to determine urinary cotinine levels by gas chromatography/mass spectrometry using a PerkinElmer Clarus 600T unit with a detection limit of $1.26 \mathrm{ng} / \mathrm{mL}$. Standard reference materials were used for internal quality assurance and control purposes (ClinChek, RECIPE, Munich, Germany). The G-EQUAS uses a 
standard protocol to measure urinary cotinine. Subjects with an urinary cotinine level of $\geq 50 \mathrm{ng} / \mathrm{mL}$ were defined as cotinine-verified smokers [21,22].

\subsection{Metabolic Syndrome}

MetS was defined as described by the American Heart Association/National Heart, Lung, and Blood Institute for clinical diagnosis and included abdominal obesity, elevated triglycerides, increased waist circumference, decreased HDL, elevated blood pressure, and elevated plasma glucose [23]. Participants with three or more of the following five risk factors were defined as having MetS: (1) elevated waist circumference (WC $\geq 80 \mathrm{~cm}$ in women and $W C \geq 90 \mathrm{~cm}$ in men), (2) elevated triglycerides (TG $\geq 150 \mathrm{mg} / \mathrm{dL}$ or receipt of medication for elevated triglycerides), (3) low high-density lipoprotein cholesterol (HDL-C $<50 \mathrm{mg} / \mathrm{dL}$ in women, HDL-C $<40 \mathrm{mg} / \mathrm{dL}$ in men or receipt of medication to increase HDL-C), (4) elevated blood pressure (systolic blood pressure $\geq 130 \mathrm{mmHg}$ and/or $\geq 85 \mathrm{mmHg}$ diastolic blood pressure or on antihypertensive drug treatment and a history of hypertension), and (5) elevated fasting glucose ( $\geq 100 \mathrm{mg} / \mathrm{dL}$ or receipt of medical treatment for elevated glucose) [23-25].

\subsection{Serum Levels of Vitamins $A, D$, and $E$}

Serum 25-hydroxyvitamin D (25(OH)D) levels were determined using a 1470 Wizard gamma counter (Perkin Elmer, Turku, Finland) and a radioimmunoassay (RIA) (DiaSorin, Stillwater, MN, USA). Vitamin D deficiency was defined as a serum $25(\mathrm{OH}) \mathrm{D}$ concentration of $<50 \mathrm{nmol} / \mathrm{L}$. Serum retinol $(\mathrm{mg} / \mathrm{L})$ and serum $\alpha$-tocopherol $(\mathrm{mg} / \mathrm{L})$ levels were measured with an Agilent1200 (Agilent1200; Agilent, Santa Clara, CA, USA) using Chromsystems (Chromsystems; Chromsystems Instruments \& Chemicals, Munich, Germany) reagents. The reference normal ranges for serum retinol and $\alpha$-tocopherol in adults are 0.30 to $0.70 \mathrm{mg} / \mathrm{L}$ and 5.00 to $20.00 \mathrm{mg} / \mathrm{L}$, respectively [20].

\subsection{Food and Vitamin Intakes}

Daily food intakes were calculated using the one-day $24 \mathrm{~h}$ recall method. Before food intakes were evaluated, all participants were instructed to maintain their normal dietary habits. Dietary intake information was also collected by administering a semi-quantitative questionnaire on food frequency, which addressed the intakes of 63 food products. Dietary intake information was completed by each participant. Food consumptions were calculated using nine categories: "never or rarely", "once a month", "two to three times a month", "one to two times a week", "three to four times a week", "five to six times a week", "once a day", "twice a day", and "three or more times every day [26]".

Three food types (green vegetables, other vegetables, and fruit) were chosen from the 63 food items. Green vegetables included spinach, cucumber, radish leaves, and pepper; other vegetables included radish, sprouts, Korean cabbage, cabbage, pumpkin, carrot, and tomato; and fruits included persimmon, tangerine, pear, watermelon, grape, peach, strawberry, apple, banana, and citrus. Subjects were divided into two groups for each food type based on food intake frequencies-that is, to low consumption ("almost never", or "less than three times a month") or high consumption groups ("2-6 times per week") [18].

The curry consumption was estimated using responses to the KNHANES food frequency questionnaire. Curried rice was the only food among the surveyed foods related to curry consumption. Subjects were divided into a low curry consumption group ("almost never", or "less than three times a month") or a high curry consumption group ("2-6 times per week") based on curry consumption frequency [27].

Daily vitamin intake was determined by summing mean $24 \mathrm{~h}$ dietary intakes using Can-Pro 3.0 nutrient intake assessment software developed by the Korean Nutrition Society. Daily total energy intakes were calculated using Korean Estimated Energy Requirements (EERs) [28]. Total vitamin A intake was measured by summing vitamin A and b-carotene intakes and dividing by 6 [18]. 


\subsection{Statistical Analysis}

The statistical analysis was performed using STATA software (version 16.0; StataCorp, College Station, TX, USA). The baseline characteristics of participants were summarized using frequencies and proportions for categorical variables, and means and standard deviations for continuous variables. Continuous and categorical variables were analyzed using the Student's $t$-test and the $\chi^{2}$ test, respectively.

Associations between MetS and related factors were examined by logistic regression. First, potential covariates were identified in the literature, based on subjective prior knowledge, or by $p$ values $\leq 0.25$ by univariate analysis, and were entered into the full model [29]. In the multivariate analysis, logistic regression models were used to identify risk factors associated with MetS; these included vitamins (mg or $\mu \mathrm{g})$, heavy metals $(\mu \mathrm{g} / \mathrm{L})$, curry consumption (low or high consumption), age ( $\leq 29,30-39,40-49,50-59, \geq 60)$, sex, residential area (rural vs. urban), marital status (married, living alone), education level ( $\leq$ middle school, high school, $\geq$ college), monthly household income $(<2000, \geq 2000$ and $<4000, \geq 4000$ and $<6000, \geq 6000$ ), smoking status (current smoker, non/ex-smoker), cotinine-verified smoker (yes, no), high-risk drinking (yes, no), physical activity (not regular, regular), BMI $(<18.5, \geq 18.5$ and $<25, \geq 25$ and $<30, \geq 30$ ), green vegetables (low or high consumption), other vegetables (low or high consumption), fruit (low or high consumption), and comorbidities (yes, no) (detailed in Supplementary Materials). To visualize the moderating effect of the MetS, marginal effects were performed using the results of logistic regression analysis. Forest plots were also produced from the models to visualize associations between MetS and risk factors. Statistical tests were two-sided, and $p$-values $<0.05$ were considered statistically significant.

\section{Results}

\subsection{Characteristics of Participants with Respect to Metabolic Syndrome}

We included 60,256 subjects that participated in the KNANES 2009-2103 and 20162017 surveys. Mean participant age was $40.8 \pm 22.8$ years (min-max: 15-80), and 32,827 $(54.5 \%)$ were women. Most subjects reported that they never or rarely consumed curry (53\%), 46\% reported that they consumed curry occasionally ("2-3 times a month" or "once a week"), while only $1 \%(67 / 10,874)$ of the subjects reported that they consumed curry often ("2-4 times a week" or "5-6 times a week"). Among the subjects with MetS $(n=21,612)$, there were significantly more females than males and more were aged $\geq 60$, unemployed, living in a rural location or alone, had a low education level, a low monthly income household, were underweight (BMI $<18.5 \mathrm{Kg} / \mathrm{m}^{2}$ ), overweight (BMI $\geq 25$ and $<30 \mathrm{Kg} / \mathrm{m}^{2}$ ), obese (BMI $\geq 30 \mathrm{Kg} / \mathrm{m}^{2}$ ), or had a family history of CVD or diabetes. Notably, the risk of MetS was significantly higher for subjects with comorbidities among those without low curry, other vegetable, or fruit consumptions. The characteristics of the study population by MetS are shown in Table 1.

Table 1. Demographic distribution of participants by metabolic syndrome.

\begin{tabular}{|c|c|c|c|c|c|}
\hline \multirow{2}{*}{ Variables } & \multirow{2}{*}{ No. } & \multicolumn{2}{|c|}{ Metabolic Syndrome } & \multirow[t]{2}{*}{ ORs 95\%CI } & \multirow[t]{2}{*}{$p$-Value } \\
\hline & & Yes & No & & \\
\hline Sex $(\%)$ & 60,256 & & & & \\
\hline Male & 27,429 & $8239(38.1)$ & $19,190(49.7)$ & 1 (refer) & \\
\hline Female & 32,827 & $13,373(61.9)$ & $19,454(50.3)$ & $1.60(1.55-1.66)$ & $<0.001$ \\
\hline Age (year) ${ }^{\dagger}$ & 60,256 & $39.6 \pm 28.0$ & $41.6 \pm 19.1$ & & $<0.001$ \\
\hline \multicolumn{6}{|l|}{ Age group (\%) } \\
\hline$<29$ & 19,626 & $8353(38.7)$ & $11,273(29.2)$ & 1 (refer) & \\
\hline $30-39$ & 8332 & $1219(5.6)$ & $7113(18.4)$ & $0.23(0.22-0.25)$ & $<0.001$ \\
\hline $40-49$ & 8656 & $1946(9.0)$ & $6710(17.4)$ & $0.39(0.37-0.41)$ & $<0.001$ \\
\hline $50-59$ & 8554 & $2861(13.2)$ & $5693(14.7)$ & $0.68(0.64-0.72)$ & $<0.001$ \\
\hline$>60$ & 15,088 & $7233(33.5)$ & $7855(20.3)$ & $1.24(1.19-1.30)$ & $<0.001$ \\
\hline Marital status (\%) & 60,126 & & & & \\
\hline
\end{tabular}


Table 1. Cont.

\begin{tabular}{|c|c|c|c|c|c|}
\hline \multirow{2}{*}{ Variables } & \multirow{2}{*}{ No. } & \multicolumn{2}{|c|}{ Metabolic Syndrome } & \multirow[t]{2}{*}{ ORs $95 \% \mathrm{CI}$} & \multirow[t]{2}{*}{$p$-Value } \\
\hline & & Yes & No & & \\
\hline Married & 39,286 & $12,929(60.0)$ & $26,357(68.3)$ & 1 (refer) & \\
\hline Living alone & 20,840 & $8611(40.0)$ & $12,229(31.7)$ & $1.44(1.39-1.49)$ & $<0.001$ \\
\hline Residential areas (\%) & 60,256 & & & & \\
\hline Urban & 48,396 & $16,919(78.3)$ & $31,477(81.4)$ & 1 (refer) & \\
\hline Rural & 11,860 & 4693 (21.7) & 7167 (18.6) & $1.22(1.17-1.27)$ & $<0.001$ \\
\hline Occupation (\%) & 44,687 & & & & \\
\hline $\begin{array}{c}\text { Managers, } \\
\text { professional }\end{array}$ & 5358 & $621(5.6)$ & $4737(14.1)$ & 1 (refer) & \\
\hline $\begin{array}{c}\text { Office worker, clerical } \\
\text { workers }\end{array}$ & 3790 & $497(4.5)$ & $3293(9.8)$ & $1.15(1.01-1.31)$ & 0.029 \\
\hline $\begin{array}{c}\text { Service workers, sales } \\
\text { workers }\end{array}$ & 5407 & $1217(11.0)$ & $4190(12.5)$ & $2.22(1.99-2.46)$ & $<0.001$ \\
\hline $\begin{array}{l}\text { Agriculture, forestry, } \\
\text { and fishing workers }\end{array}$ & 2848 & $900(8.1)$ & $1948(5.8)$ & $3.52(3.14-3.95)$ & $<0.001$ \\
\hline $\begin{array}{c}\text { Craft, plant, and } \\
\text { machine operators and } \\
\text { assemblers }\end{array}$ & 4029 & $677(6.1)$ & $3352(10.0)$ & $1.54(1.37-1.73)$ & $<0.001$ \\
\hline $\begin{array}{l}\text { Elementary } \\
\text { occupations }\end{array}$ & 3730 & $1111(10.1)$ & $2619(7.7)$ & $3.24(2.90-3.61)$ & $<0.001$ \\
\hline Unemployed & 19,525 & $6036(54.6)$ & $13,489(40.1)$ & $3.41(3.12-3.73)$ & $<0.001$ \\
\hline Education level (\%) & 55,326 & & & & \\
\hline$\leq$ Middle school & 27,702 & $13,669(76.4)$ & $14,033(37.5)$ & 1 (refer) & \\
\hline High school & 14,342 & $2689(15.0)$ & $11,653(31.1)$ & $0.24(0.23-0.25)$ & $<0.001$ \\
\hline$\geq$ College & 13,282 & $1544(8.6)$ & $11,738(21.4)$ & $0.14(0.13-0.14)$ & $<0.001$ \\
\hline $\begin{array}{l}\text { Monthly household } \\
\text { income }(\%)^{\S}\end{array}$ & 59,628 & & & & \\
\hline$<2000$ & 16,917 & $7264(34.0)$ & $9653(25.2)$ & 1 (refer) & \\
\hline$\geq 2000$ and $<4000$ & 19,423 & $6922(32.4)$ & $12,501(32.7)$ & $0.74(0.71-0.77)$ & $<0.001$ \\
\hline$\geq 4000$ and $<6000$ & 13,065 & $4176(19.6)$ & $8889(23.2)$ & $0.62(0.60-0.65)$ & $<0.001$ \\
\hline$\geq 6000$ & 10,223 & $2982(14.0)$ & 7241 (18.9) & $0.55(0.52-0.58)$ & $<0.001$ \\
\hline BMI group (\%) & 56,009 & & & & \\
\hline$<18.5$ & 8945 & $5057(29.0)$ & 3888 (10.1) & $5.71(5.43-6.01)$ & $<0.001$ \\
\hline$\geq 18.5$ and $<25$ & 31,942 & 5925 (33.9) & $26,017(67.5)$ & 1 (refer) & \\
\hline$\geq 25$ and $<30$ & 13,096 & $5337(30.6)$ & 7759 (20.1) & $3.02(2.89-3.16)$ & $<0.001$ \\
\hline$\geq 30$ & 2026 & $1137(6.5)$ & $889(2.3)$ & $5.62(5.12-6.16)$ & $<0.001$ \\
\hline $\begin{array}{c}\text { Curry consumption } \\
(\%)\end{array}$ & 10,874 & & & & \\
\hline Rarely or never & 5812 & $1587(61.9)$ & $4225(50.8)$ & 1 (refer) & \\
\hline Often and occasionally & 5062 & $977(38.1)$ & 4085 (49.2) & $0.64(0.58-0.70)$ & $<0.001$ \\
\hline $\begin{array}{l}\text { Green vegetable } \\
\text { consumption }(\%)\end{array}$ & 10,874 & & & & \\
\hline Rarely or never & 5838 & $1419(55.3)$ & 4419 (53.2) & 1 (refer) & \\
\hline Often and occasionally & 5036 & $1145(44.7)$ & $3891(46.8)$ & $0.92(0.84-1.00)$ & 0.055 \\
\hline $\begin{array}{l}\text { Other vegetable } \\
\text { consumption }(\%)\end{array}$ & 10,914 & & & & \\
\hline Rarely or never & 7468 & $1809(70.2)$ & $5659(67.9)$ & 1 (refer) & \\
\hline Often and occasionally & 3446 & $769(29.8)$ & $2677(32.1)$ & $0.90(0.82-0.99)$ & 0.029 \\
\hline Fruit (\%) & 10,916 & & & & \\
\hline Rarely or never & 6691 & $1726(66.9)$ & 4965 (59.5) & 1 (refer) & \\
\hline Often and occasionally & 4225 & $852(33.1)$ & $3373(30.5)$ & $0.73(0.66-0.80)$ & $<0.001$ \\
\hline $\begin{array}{l}\text { Cotinine verified } \\
\text { smoker }(\%)\end{array}$ & 60,256 & & & & \\
\hline No & 16,780 & $4468(20.7)$ & $12,312(31.9)$ & 1 (refer) & \\
\hline Yes & 43,476 & $17,144(79.3)$ & $26,332(68.1)$ & $1.79(1.73-1.89)$ & $<0.001$ \\
\hline Smoking status (\%) & 42,803 & & & & \\
\hline Non-/ex-smoker & 32,992 & $9413(84.5)$ & $23,579(74.5)$ & 1 (refer) & \\
\hline
\end{tabular}


Table 1. Cont.

\begin{tabular}{|c|c|c|c|c|c|}
\hline \multirow{2}{*}{ Variables } & \multirow{2}{*}{ No. } & \multicolumn{2}{|c|}{ Metabolic Syndrome } & \multirow[t]{2}{*}{ ORs $95 \% \mathrm{CI}$} & \multirow[t]{2}{*}{$p$-Value } \\
\hline & & Yes & No & & \\
\hline Current smoker & 9811 & $1728(15.5)$ & $8083(25.5)$ & $0.54(0.51-0.57)$ & $<0.001$ \\
\hline Drinking status (\%) & 47,435 & & & & \\
\hline Often & 15,884 & $4814(42.8)$ & $11,070(30.6)$ & 1 (refer) & \\
\hline Occasionally & 22,403 & $4511(40.1)$ & $17,892(49.4)$ & $0.58(0.55-0.61)$ & $<0.001$ \\
\hline Never or rarely & 9148 & $1915(17.0)$ & $7233(20.0)$ & $0.61(0.57-0.65)$ & $<0.001$ \\
\hline Physical activity (\%) & 60,256 & & & & \\
\hline Not regular & 51,088 & $19,677(91.1)$ & $31,411(81.3)$ & 1 (refer) & \\
\hline Regular & 9168 & $1935(8.9)$ & $7233(18.7)$ & $0.43(0.40-0.45)$ & $<0.001$ \\
\hline $\begin{array}{c}\text { Family history of } \\
\text { CVDs (\%) }\end{array}$ & 60,256 & & & & \\
\hline No & 51,088 & $4337(50.0)$ & $17,727(60.2)$ & 1 (refer) & \\
\hline Yes & 9168 & $4331(50.0)$ & $11,703(39.8)$ & $1.51(1.44-1.59)$ & $<0.001$ \\
\hline $\begin{array}{c}\text { Family history of } \\
\text { diabetes (\%) }\end{array}$ & 37,720 & & & & \\
\hline No & 30,335 & $6375(75.5)$ & $23,960(81.8)$ & 1 (refer) & \\
\hline Yes & 7385 & $2071(24.5)$ & $5314(18.2)$ & $1.46(1.38-1.55)$ & $<0.001$ \\
\hline $\begin{array}{c}\text { Family history of } \\
\text { hyperlipidemia (\%) }\end{array}$ & 36,414 & & & & \\
\hline No & 34,145 & 7525 (94.2) & $26,620(93.6)$ & 1 (refer) & \\
\hline Yes & 2269 & $461(5.8)$ & $1808(6.4)$ & $0.90(0.81-1.00)$ & 0.055 \\
\hline Comorbidities $\mathbb{I}$ & & & & & \\
\hline $\begin{array}{l}\text { Type } 2 \text { diabetes } \\
\text { mellitus }\end{array}$ & 3793 & $2525(13.9)$ & $1268(3.3)$ & $4.67(4.35-5.01)$ & $<0.001$ \\
\hline Hypertension & 9837 & 5927 (32.6) & $3910(10.3)$ & $4.21(4.03-4.41)$ & $<0.001$ \\
\hline Dyslipidemia & 5532 & $3332(32.8)$ & $2200(6.0)$ & $7.59(7.15-8.06)$ & $<0.001$ \\
\hline Stroke & 906 & $486(2.7)$ & $420(1.12)$ & $2.45(2.15-2.80)$ & $<0.001$ \\
\hline MI or angina & 1130 & $565(3.4)$ & $565(1.6)$ & $2.21(1.96-2.48)$ & $<0.001$ \\
\hline MI & 393 & $178(1.0)$ & $215(0.6)$ & $1.73(1.42-2.12)$ & $<0.001$ \\
\hline Angina & 810 & $421(2.3)$ & $389(1.0)$ & $2.29(1.99-2.63)$ & $<0.001$ \\
\hline Asthma & 1965 & $804(4.5)$ & $1161(3.1)$ & $1.47(1.34-1.61)$ & $<0.001$ \\
\hline Thyroid disease & 1607 & $585(3.3)$ & $1022(2.7)$ & $1.20(1.08-1.33)$ & 0.001 \\
\hline Osteoarthritis & 4984 & $2706(15.1)$ & $2278(6.1)$ & $2.74(2.58-2.91)$ & $<0.001$ \\
\hline Rheumatoid arthritis & 880 & $378(2.1)$ & $502(1.3)$ & $1.58(1.38-1.81)$ & $<0.001$ \\
\hline Arthritis & 5628 & $2948(17.5)$ & $2680(7.3)$ & $2.68(2.53-2.83)$ & $<0.001$ \\
\hline Kidney failure & 178 & $89(0.5)$ & $89(0.2)$ & $2.09(1.56-2.81)$ & $<0.001$ \\
\hline Depression & 1785 & $706(3.9)$ & $1079(2.9)$ & $1.38(1.25-1.52)$ & $<0.001$ \\
\hline $\begin{array}{l}\text { Waist circumference } \\
\qquad(\mathrm{cm})^{+}\end{array}$ & 56,935 & $77.6 \pm 10.5$ & $74.2 \pm 19.0$ & - & $<0.001$ \\
\hline $\begin{array}{l}\text { Total cholesterol } \\
(\mathrm{mg} / \mathrm{dL})^{+}\end{array}$ & 47,054 & $195.9 \pm 40.9$ & $183.8 \pm 35.6$ & - & $<0.001$ \\
\hline $\mathrm{LDL}_{-\mathrm{C}}(\mathrm{mg} / \mathrm{dL})^{+}$ & 10,339 & $117.4 \pm 36.3$ & $111.3 \pm 31.9$ & - & $<0.001$ \\
\hline Triglyceride (mg/dL) & 47,054 & $194.7 \pm 136.4$ & $110.9 \pm 87.7$ & - & $<0.001$ \\
\hline $\mathrm{HDL}-\mathrm{C}(\mathrm{mg} / \mathrm{dL})^{\dagger}$ & 47,054 & $44.3 \pm 9.8$ & $51.6 \pm 12.0$ & - & $<0.001$ \\
\hline $\operatorname{HbA1c}(\%)^{+}$ & 33,118 & $6.4 \pm 1.2$ & $5.6 \pm 0.7$ & - & $<0.001$ \\
\hline $\begin{array}{l}\text { Fasting glucose } \\
(\mathrm{mg} / \mathrm{dL})^{+}\end{array}$ & 46,984 & $113.7 \pm 32.0$ & $93.8 \pm 16.6$ & - & $<0.001$ \\
\hline Energy intake $(\mathrm{Kcal})^{\dagger}$ & 53,701 & $1680.9 \pm 797.4$ & $2026.6 \pm 880.2$ & - & $<0.001$ \\
\hline Hemoglobin $(\mathrm{g} / \mathrm{dL})^{+}$ & 46,846 & $13.7 \pm 1.5$ & $14.0 \pm 1.6$ & - & $<0.001$ \\
\hline $\operatorname{ALT}(\mathrm{U} / \mathrm{L})^{+}$ & 47,054 & $24.9 \pm 17.9$ & $19.7 \pm 17.7$ & - & $<0.001$ \\
\hline $\operatorname{AST}(\mathrm{U} / \mathrm{L})^{+}$ & 47,054 & $24.8 \pm 12.9$ & $21.3 \pm 13.1$ & - & $<0.001$ \\
\hline $\begin{array}{l}\text { Systolic blood pressure } \\
(\mathrm{mmHg})^{+}\end{array}$ & 50,220 & $130.3 \pm 17.4$ & $114.4 \pm 15.2$ & - & $<0.001$ \\
\hline $\begin{array}{l}\text { Diastolic blood } \\
\text { pressure }(\mathrm{mmHg})\end{array}$ & 50,220 & $78.8 \pm 11.4$ & $73.6 \pm 10.4$ & - & $<0.001$ \\
\hline
\end{tabular}

${ }^{\dagger}:$ means \pm SDs, two-sample $t$-test with unequal variances, except for ALT with equal variances; ${ }^{\mathbb{I}}$ : reference with no comorbidities;

$\S$ : thousand won. AST: aspartate aminotransferase. ALT: alanine aminotransferase, MI: myocardial infarction. BMI: body mass index $\left(\mathrm{kg} / \mathrm{m}^{2}\right)$, CVD: cardiovascular disease, HDL: high-density lipoprotein, LDL-C: low-density lipoprotein cholesterol. 


\subsection{Characteristics of Daily Vitamin Intakes and Serum Heavy Metal Levels}

Average daily intakes of vitamin B1, B2, B3, and C were $1.35 \pm 0.86 \mathrm{mg}(95 \% \mathrm{CI}$ $1.34-1.36) ; 1.28 \pm 0.82 \mathrm{mg}$ (95\% CI 1.28-1.29); $14.64 \pm 9.08 \mathrm{mg}$ (95\% CI 14.56-14.72); and $90.10 \pm 95.76 \mathrm{mg}(95 \% \mathrm{CI} 89.30-90.90)$, respectively. Average daily intakes of total vitamin A (mean \pm SE) and retinol were $692.12 \pm 4.44 \mu \mathrm{g}$ (95\% CI 683.43-700.81) and $125.36 \pm 345.14 \mu \mathrm{g}$ (95\% CI 122.44-128.28), respectively. Average levels of serum $\mathrm{Pb}, \mathrm{Hg}$, $\mathrm{Cd}$, and hs-CRP were $2.06 \pm 1.10 \mu \mathrm{g} / \mathrm{dL}$ (95\% CI 2.04-2.07); $4.08 \pm 3.53 \mu \mathrm{g} / \mathrm{L}$ (95\% CI $4.02-4.13) ; 1.02 \pm 0.67 \mu \mathrm{g} / \mathrm{L}$ (95\% CI 1.01-1.03), and $1.20 \pm 2.02 \mathrm{mg} / \mathrm{L}$ (95\% CI 1.171.24), respectively. No sample had a value of below an LOD. Serum vitamin $A, E$, and D concentrations were $0.54 \pm 0.19 \mathrm{mg} / \mathrm{L}(95 \%$ CI $0.53-0.55), 13.72 \pm 6.35 \mathrm{mg} / \mathrm{L}(95 \%$ CI 13.43 14.00), and $17.54 \pm 6.16 \mathrm{nmol} / \mathrm{L}$ (95\% CI 17.47-17.60), respectively. Vitamin B1 (1.24 \pm 0.82 vs. $1.44 \pm 0.87, p<0.001)$, B2 (1.17 \pm 0.76 vs. $1.36 \pm 0.84, p<0.001)$, B3 (13.08 \pm 8.61 vs. $15.81 \pm 9.25, p<0.001), \mathrm{C}(83.03 \pm 87.46$ vs. $95.38 \pm 101.23, p<0.001)$, and $\mathrm{A}(628.67 \pm 6.78$ vs. $740.24 \pm 5.86, p<0.001)$ intakes were significantly lower in subjects with than without MetS. On the other hand, serum levels of $\mathrm{Pb}(2.34 \pm 1.22$ vs. $1.96 \pm 1.03, p<0.001), \mathrm{Hg}$ $(4.78 \pm 3.87$ vs. $3.83 \pm 3.37, p<0.001)$, Cd ( $1.26 \pm 0.69$ vs. $0.93 \pm 0.64, p<0.001)$, vitamin A $(0.61 \pm 0.21$ vs. $0.51 \pm 0.18, p<0.001), \mathrm{E}(15.78 \pm 8.33$ vs. $12.95 \pm 5.23, p<0.001)$, and hs-CRP $(1.58 \pm 2.33$ vs. $1.02 \pm 1.83, p<0.001)$ were significantly higher in subjects with MetS.

After adjustment for comorbidities, the risk of MetS was found to be significantly lower for subjects with high curry consumption than subjects with low curry consumption (OR $0.64 ; 95 \%$ CI, $0.58-0.70, p<0.001$ ). Furthermore, risks of MetS were reduced by $44 \%$ (OR 0.56; 95\% CI, 0.54-0.58, $p<0.001$ ), 38\% (OR 0.62; 95\% CI, $0.61-0.64, p<0.001$ ), $7 \%$ (OR 0.93; 95\% CI, 0.92-0.93, $p<0.001$ ), and 1\% (OR 0.99; 95\% CI, $0.98-0.99, p<0.001$ ), when vitamin B1, B2, B3, and C intakes increased by one $\mathrm{mg}$ daily, respectively. However, risks of MetS were increased by 19\% (OR 1.19; 95\% CI, 1.15-1.24, $p<0.001$ ), 3\% (OR 1.03; $95 \%$ CI, 1.02-1.05, $p<0.001$ ), 113\% (OR 2.13; 95\% CI, 2.00-2.27, $p<0.001$ ), and $6 \%$ (OR 1.06; 95\% CI, 1.03-1.09, $p<0.001$ ), when serum levels of $\mathrm{Pb}, \mathrm{Hg}$, Cd, and hs-CRP increased by one unit.

After adjustment for potential confounders including monthly household income, residential area, energy intake, occupation, sex, family history of CVDs, family history of diabetes mellites, family history of hyperlipidemia, BMI, smoking status, cotinine verified smoker, high-risk drinking, physical activity, education level, hypertension, dyslipidemia, type 2 diabetes, stroke, myocardial infarction or angina, myocardial infarction, angina, arthritis, osteoarthritis, rheumatoid arthritis, kidney failure, depression, thyroid disease, asthma, and green vegetable, other vegetable, and fruit consumption (details are shown in Supplementary Materials), adjusted odds ratios followed a similar pattern. The risk of MetS was significantly lower for high than low curry consumers (OR $0.85 ; 95 \% \mathrm{CI}$, $0.74-0.98, p=0.028)$ and reduced by $12 \%$ (OR $0.88 ; 95 \% \mathrm{CI}, 0.79-0.97, p=0.013)$ and $1 \%$ (OR 0.99; 95\% CI, 0.98-0.99, $p=0.032$ ) when vitamin B1 and C intakes increased by one mg, respectively. Similarly, risks of MetS were increased by $14 \%$ (OR 1.14; 95\% CI, $1.03-1.28, p=0.015), 3 \%(\mathrm{OR} 1.03 ; 95 \% \mathrm{CI}, 1.02-1.06, p=0.031)$, and 9\% (OR 1.09; 95\% CI, $1.04-1.14, p=0.001)$, when serum $\mathrm{Pb}, \mathrm{Hg}$, and hs-CRP levels increased by one unit. Crude odds ratios and adjusted odds ratios (95\% confidence interval) for risks of MetS are shown in Figure 1.

Figure 2 shows the marginal effects of vitamin intakes, curry consumption, serum heavy metals, and hs-CRP on MetS by age group in the Korean population after adjustment for potential cofounders. The effects of vitamin and curry intakes showed similar trends. The probability of MetS decreased when vitamin or curry intakes increased but increased when serum $\mathrm{Pb}, \mathrm{Hg}$, or hs-CRP levels increased. 


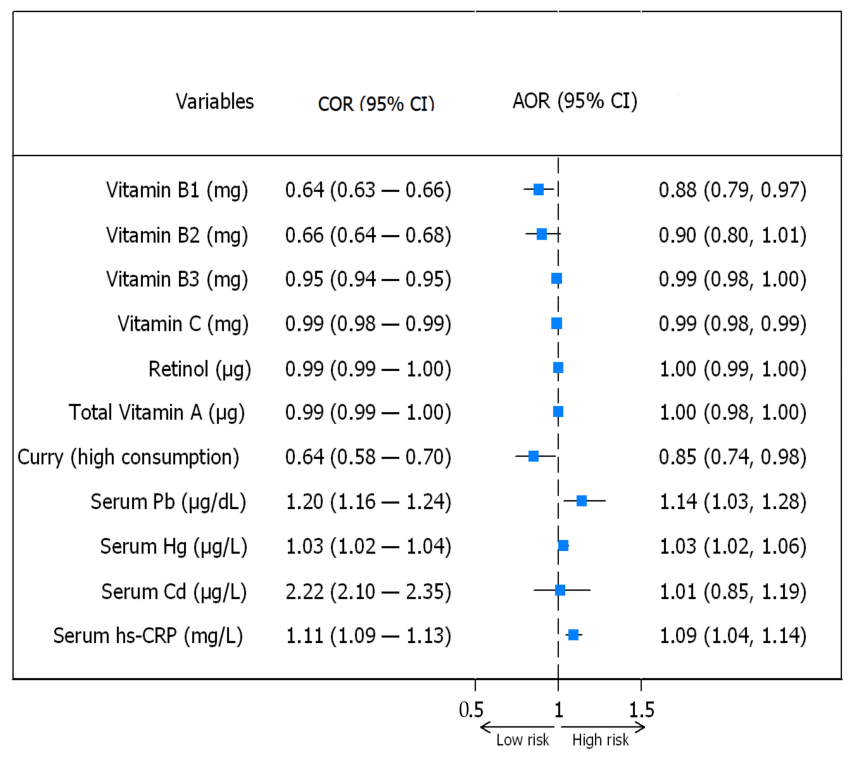

Figure 1. Crude odds ratio and adjusted odds ratio (95\% confidence interval) for risks of MetS.

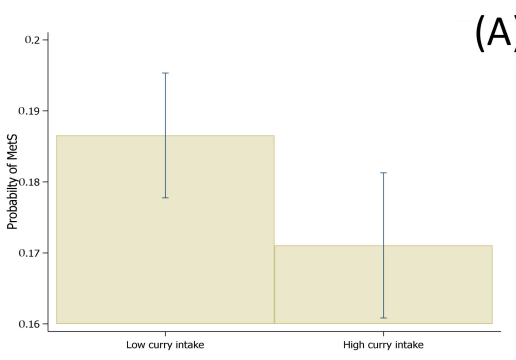

(B)
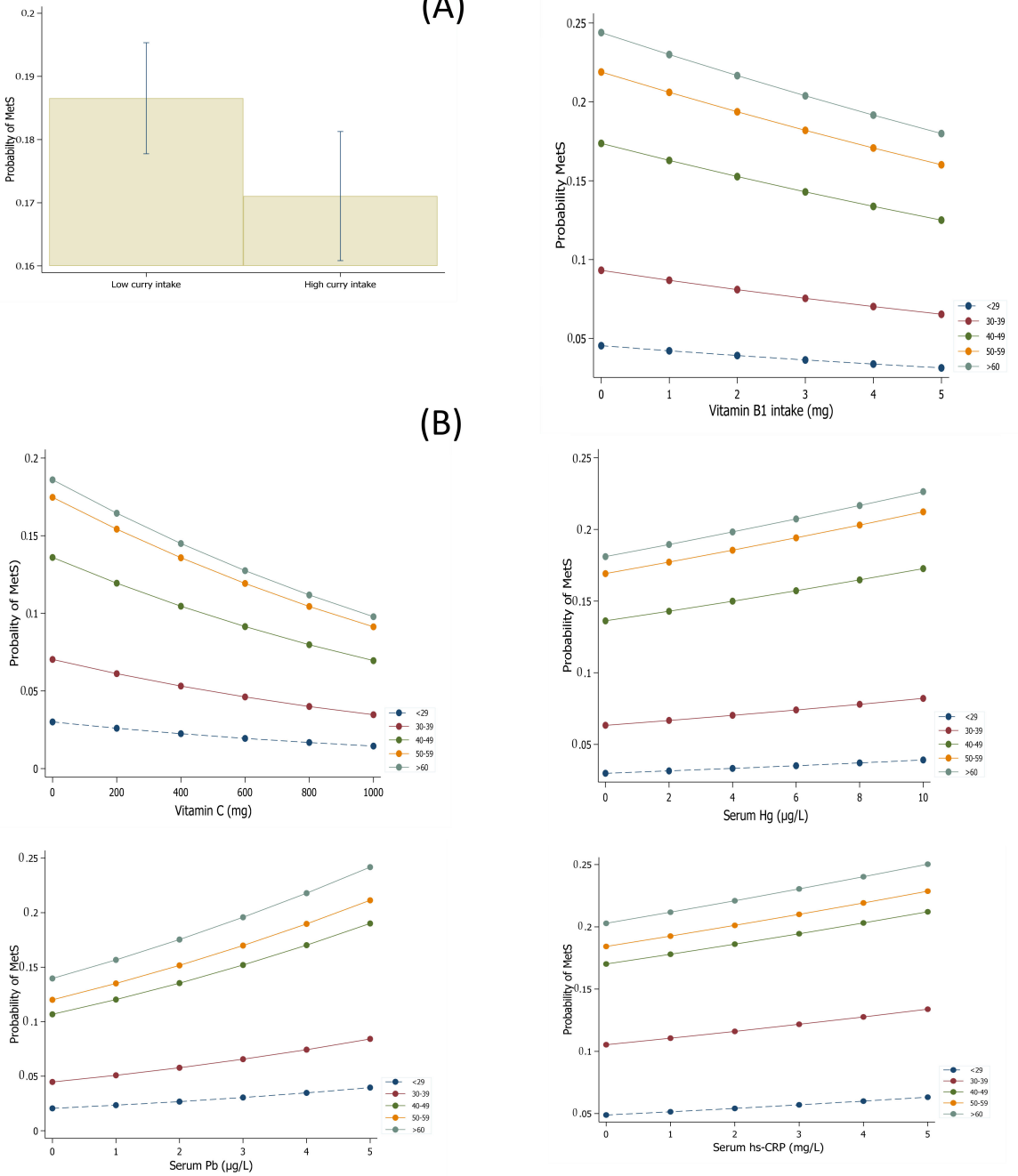

Figure 2. Marginal effects of vitamin intakes, curry consumption (A), and serum levels of heavy metals and hs-CRP (B) on MetS by age group in the Korean population after adjustment for potential cofounders. 


\section{Discussion}

Our epidemiological findings provide evidence that supports experimental knowledge regarding associations between vitamin intake, curry consumption, and heavy metal exposure and MetS and subjects with MetS and comorbidities. We identified associations between MetS and vitamin intakes, serum heavy metal levels, hs-CRP, and comorbidities in a cohort representative of the Korean population. Vitamin intakes and high curry consumption exhibited inverse correlations with the prevalence of MetS, while serum levels of heavy metals and hs-CRP were positively correlated with MetS.

Dramatic global increases in urbanization and industrialization have increased the risk of exposure to pollutants, especially heavy metals [30]. The growing global burden posed by NCDs has made their prevention and management a priority, and this is especially true in the context of the COVID-19 pandemic because COVID-19 is associated with NCD-induced morbidity and mortality [31].

In this study, we found that levels of serum heavy metals and cardiometabolic risk factors were positively correlated with MetS, which agrees with the results of a previous study $[9,32]$. Heavy metals such as $\mathrm{Pb}, \mathrm{Hg}$, and $\mathrm{Cd}$ are toxic and can trigger various diseases, especially CVDs [33]. In addition, they can increase ROS and reactive nitrogen species levels and cause oxidative stress, DNA damage, and the oxidation of protein thiol groups [34]. Furthermore, heavy metals provoke the production of inflammatory cytokines and anti-thrombotic agents [6,7]. Recent data also indicate that elevated hs-CRP levels increase the risk of MetS development in obese and non-obese women [35]. These findings indicate that the harmful impacts of multiple environmental pollutants, including heavy metals, on MetS should be urgently addressed and that a preventative strategy targeting the high-risk population is required to reduce the negative impacts of environmental pollutants and heavy metals.

Vitamin B1 plays an important role in intracellular glucose metabolism by acting as a coenzyme for $\alpha$-ketoglutarate dehydrogenase complexes, transketolase, and pyruvate dehydrogenase [36], and our study shows that vitamin B1 intakes were significantly lower and that levels of $\mathrm{HbA} 1 \mathrm{c}$ and fasting glucose were significantly higher in individuals with MetS. It has been shown that reduced vitamin B1 levels in diabetic vascular cells exacerbate metabolic dysfunction in hyperglycemia [37], and genetic studies have reported that the relationship between diabetes and vitamin B1 is associated with transketolase (Tk), $\alpha-1$ antitrypsin, the SLC19A2 gene, and p53 [38-41]. Vitamin B1 and its derivatives may hinder the biochemical pathways leading to caspase activation, for example, by increased flux via the polyol or hexosamine biosynthesis pathways, inducing the production of advanced glycation end-products or activation of protein kinase C [42-45]. Our findings are in line with those of a previous study, in which thiamine at $150 \mathrm{mg}$ daily for 1 month significantly reduced plasma fasting glucose concentrations in patients with drug-naïve type 2 diabetes [46]. The consumption of vitamin B1 has also been reported to be inversely related to dyslipidemia [47]. Vitamin B1 attenuates the adverse consequences of high endothelial glucose levels by reducing the glycation of intracellular proteins [48], plays a vital role in the prevention of atherosclerotic plaque, and inhibits the glucose and insulin-mediated proliferation of human infragenicular arterial smooth muscle cells [49]. Several studies have shown that regular vitamin B1 administration increases the functions of endothelial cells and retards the development of atherosclerosis [50], and short-term vitamin B1 treatment regenerated endothelial cell functions in otherwise healthy smokers with endothelial dysfunction caused by smoking [51].

Oxidative stress could play a significant part in the etiology of MetS [52], and MetS patients exhibit systemic oxidative damage due to ROS upregulation and/or reduced levels of antioxidant enzymes [53]. Although our findings show that regular vitamin B1 and $\mathrm{C}$ intake reduces the risk of MetS, other studies have reported associations between vitamin B2, B3, A, E, and MetS $[18,54,55]$. It has been reported that vitamin B2 prevents pro-inflammatory activity in adipocyte and macrophage co-cultures and thus might reduce the mild inflammation associated with obesity [54]. Another study showed that vitamin B2 
deficiency might increase the pro-inflammatory activities of adipocyte cells and result in chronic inflammation in the obese [56]. Vitamin B3 has also been found to be an efficient antioxidant that reduces ROS production and prevents DNA damage in lymphocytes [57], which is in line with the observation that vitamin B3 treatment promoted the normalization of low HDL-C atherogenic dyslipidemia [58]. Furthermore, a negative correlation was reported between diets enriched with specific antioxidants, such as vitamin C, A, and E, and oxidative stress [59]. These findings show that efforts are required to establish targeted vitamin B1 and C intakes in Korea. We believe these strategies would effectively diminish the prevalence of MetS.

Our analyses revealed that the risk of MetS was significantly lower among high curry consumers, which supports our hypothesis that high curry consumption reduces the risk of type 2 diabetes and the findings of previous studies [60]. Curcumin could improve endothelial function and reduce oxidative stress and levels of inflammatory markers (IL-6, TNF alpha, endothelin-1) in type 2 diabetes patients and enhance the functions of $\beta$ cells. [61,62]. Of note, curcumin also has an impact on insulin secretion in healthy subjects [63], and in patients with acute coronary syndrome, curcumin reduced triglyceride, total cholesterol, and LDL-C levels but increased HDL-C in [64]. Furthermore, recent data indicate that curcumin has a preventive effect on stroke by reducing oxidative stress related to signaling the uncoupling of protein 2, thus strengthening endothelial vascular function [65]. Curcumin also has a profound effect on microglial response, facilitates microglial M2 polarization, and prevents pro-inflammatory responses by microglia. Additionally, in ischemic stroke patients, curcumin post-treatment diminished brain damage and strengthened vascular endothelial function [66]. These data demonstrate the potential benefits of curcumin for diabetes and CVDs and show that curcumin is not only a promising alternative therapeutic for type 2 diabetes but also offers a preventive strategy for CVDs.

To the best of our knowledge, this large-scale study is the first to report the combined effects of vitamins, curry consumption, and serum heavy metal and hs-CRP levels on MetS at a national level in Korea. However, the study has several limitations. First, the cross-sectional design of the KNHANES studies prevented our evaluation of causality between MetS and these factors. Second, as no physiological markers of antioxidant status were measured, oxidation status and vitamin levels in serum (such as B vitamins, vitamin C), vitamin E intakes of food source, and tissues were not evaluated. Third, consumptions of food and vitamins were measured using one-day $24 \mathrm{~h}$ recall data and thus may have been under- or overestimated. However, all participants were instructed to maintain their usual dietary habits and the one-day $24 \mathrm{~h}$ recall method is commonly used to assess food intake. Finally, relatively few individuals consumed curry frequently, which may have influenced results regarding the association between curry consumption and MetS.

\section{Conclusions}

The prevalence of MetS and heavy metal exposure in Korea show increasing trends [67-69], and these have worsened during the COVID-19 pandemic [70]. MetS is a significant risk factor of severe COVID-19, but the mechanisms involved have yet to be determined. Dramatic increases in urbanization and industrialization have elevated risks of heavy metal exposure, and it is critical that a prevention strategy targeting the high-risk population be devised to reverse this trend. Vitamins and curry supplements might reduce the prevalence of MetS, and our results indicate that thiamine and curry intakes might protect the public against the dual burden of communicable and non-communicable diseases in Korea. However, further work is required to determine the nature of the mechanisms involved.

Supplementary Materials: The following are available online at https://www.mdpi.com/article/10 .3390 / antiox10050808/s1, Table S1. Adjusted ORs for serum lead (Pb), Table S2. Adjusted ORs for serum mercury (Hg), Table S3. Adjusted ORs for serum c-reactive protein (CRP), Table S4. Adjusted ORs for vitamin B1 intake, Table S5. Adjusted ORs for vitamin C intake, Table S6. Adjusted ORs for curry consumption. 
Author Contributions: Study concept and design (H.N.D. and M.-S.K.); acquisition of data (H.N.D. and M.-S.K.); analysis and interpretation of data (H.N.D., H.O. and M.-S.K.); statistical analysis (H.N.D. and M.-S.K.), drafting of the manuscript (H.N.D. and M.-S.K.). All authors have read and agreed to the published version of the manuscript.

Funding: This study was supported by the National Research Foundation of Korea (NRF) funded by the Korean government (MEST) (grant nos. NRF2013R1A1A3008851 and 2018R1D1A1B07049610).

Institutional Review Board Statement: This study was approved by the KNHANES inquiry commission and the Institutional Review Board of Sunchon National University in accord with as the Declaration of Helsinki.

Informed Consent Statement: Informed consent was obtained from all subjects involved in the study.

Acknowledgments: The authors are grateful to all research staff involved in the data collection required for this study.

Conflicts of Interest: The authors have no conflict of interest to declare.

\section{References}

1. WHO. Diet, Nutrition, and the Prevention of Chronic Diseases: Report of a Joint WHO/FAO Expert Consultation; World Health Organization: Geneva, Switzerland, 2003; Volume 916.

2. Song, S.; Song, H. Dietary and lifestyle factors associated with weight status among Korean adolescents from multicultural families: Using data from the 2017-2018 Korea youth risk behavior surveys. Korean J. Community Nutr. 2019, 24, 465-475. [CrossRef]

3. Huang, P.L. A comprehensive definition for metabolic syndrome. Dis. Models Mech. 2009, 2, 231-237. [CrossRef]

4. Costa, F.F.; Rosário, W.R.; Farias, A.C.R.; de Souza, R.G.; Gondim, R.S.D.; Barroso, W.A. Metabolic syndrome and COVID-19: An update on the associated comorbidities and proposed therapies. Diabetes Metab. Syndr. Clin. Res. Rev. 2020, 14, 809-814. [CrossRef]

5. Le Roux, C.W. COVID-19 alters thinking and management in metabolic diseases. Nat. Rev. Endocrinol. 2021, 17, 71-72. [CrossRef]

6. Arbi, S.; Oberholzer, H.M.; Van Rooy, M.J.; Venter, C.; Bester, M.J. Effects of chronic exposure to mercury and cadmium alone and in combination on the coagulation system of Sprague-Dawley rats. Ultrastruct. Pathol. 2017, 41, 275-283. [CrossRef] [PubMed]

7. Angeli, J.K.; Pereira, C.A.C.; de Oliveira Faria, T.; Stefanon, I.; Padilha, A.S.; Vassallo, D.V. Cadmium exposure induces vascular injury due to endothelial oxidative stress: The role of local angiotensin II and COX-2. Free Radic. Biol. Med. 2013, 65, 838-848. [CrossRef] [PubMed]

8. Valera, B.; Muckle, G.; Poirier, P.; Jacobson, S.W.; Jacobson, J.L.; Dewailly, E. Cardiac autonomic activity and blood pressure among Inuit children exposed to mercury. Neurotoxicology 2012, 33, 1067-1074. [CrossRef] [PubMed]

9. Poursafa, P.; Ataee, E.; Motlagh, M.E.; Ardalan, G.; Tajadini, M.H.; Yazdi, M.; Kelishadi, R. Association of serum lead and mercury level with cardiometabolic risk factors and liver enzymes in a nationally representative sample of adolescents: The CASPIAN-III study. Environ. Sci. Pollut. Res. 2014, 21, 13496-13502. [CrossRef]

10. Kim, N.H.; Hyun, Y.Y.; Lee, K.-B.; Chang, Y.; Ryu, S.; Oh, K.-H.; Ahn, C. Erratum: Environmental heavy metal exposure and chronic kidney disease in the general population. J. Korean Med Sci. 2015, 30, 507. [CrossRef]

11. Yamamoto, C.; Kaji, T.; Sakamoto, M.; Kozuka, H. Cadmium stimulation of plasminogen activator inhibitor-1 release from human vascular endothelial cells in culture. Toxicology 1993, 83, 215-223. [CrossRef]

12. Vaziri, N.D. Mechanisms of lead-induced hypertension and cardiovascular disease. Am. J. Physiol. Heart Circ. Physiol. 2008, 295, H454-H465. [CrossRef] [PubMed]

13. Salonen, J.T.; Seppänen, K.; Lakka, T.A.; Salonen, R.; Kaplan, G.A. Mercury accumulation and accelerated progression of carotid atherosclerosis: A population-based prospective 4-year follow-up study in men in eastern Finland. Atherosclerosis 2000, 148, 265-273. [CrossRef]

14. Eshak, E.; Arafa, A. Thiamine deficiency and cardiovascular disorders. Nutr. Metab. Cardiovasc. Dis. 2018, 28, 965-972. [CrossRef]

15. Kunnumakkara, A.B.; Bordoloi, D.; Padmavathi, G.; Monisha, J.; Roy, N.K.; Prasad, S.; Aggarwal, B.B. Curcumin, the golden nutraceutical: Multitargeting for multiple chronic diseases. Br. J. Pharmacol. 2017, 174, 1325-1348. [CrossRef] [PubMed]

16. Korea Centers for Disease Control and Prevention. Korea National Health \& Nutrition Examination Survey. Available online: https:/ / knhanes.cdc.go.kr/knhanes/eng/index.do (accessed on 20 January 2021).

17. An, J.Y.; Hong, Y.R.; Kong, S.G.J.C.; Pediatrics, E. Changes in the prevalence of anemia in Korean adolescents, 1998-2018. Clin. Exp. Pediatrics 2021, 64, 86. [CrossRef]

18. Park, S.; Ham, J.-O.; Lee, B.-K. Effects of total vitamin A, vitamin C, and fruit intake on risk for metabolic syndrome in Korean women and men. Nutrition 2015, 31, 111-118. [CrossRef]

19. Lee, K.-J.; Kim, J.-I. Relating factors for depression in Korean working women: Secondary analysis of the fifth Korean National Health and Nutrition Examination Survey (KNHANES V). Asian Nurs. Res. 2015, 9, 265-270. [CrossRef] [PubMed] 
20. Hong, K.-H.; Lee, Y. Negative correlation between vitamin A and positive correlation between vitamin E and inflammation among healthy adults in Korea: Based on the Korea National Health and Nutrition Examination Survey (KNHANES) 2016-2018 7th Edition. J. Inflamm. Res. 2020, 13, 799. [CrossRef]

21. Benowitz, N.; Jacob, P.; Ahijevych, K.; Jarvis, M.; Hall, S.; LeHouezec, J. SRNT subcommittee on biochemical verification. Biochem. Verif. Tob. Use Cessat. Nicotine Tob. Res. 2002, 4, 149-159. [CrossRef]

22. Jung-Choi, K.-H.; Khang, Y.-H.; Cho, H.-J. Hidden female smokers in Asia: A comparison of self-reported with cotinine-verified smoking prevalence rates in representative national data from an Asian population. Tob. Control 2012, 21, 536-542. [CrossRef]

23. Gregory, C.O.; McCullough, M.L.; Ramirez-Zea, M.; Stein, A.D. Diet scores and cardio-metabolic risk factors among Guatemalan young adults. Br. J. Nutr. 2008, 101, 1805-1811. [CrossRef]

24. World Health Organization. Western pacific region, International association for the study of obesity. The Asia-pacific perspective. Redefining Obes. Its Treat., 2000. Available online: https://apps.who.int/iris/handle/10665/206936 (accessed on 17 December 2020).

25. Yun, S.; Duc, H.N.; Park, J.S.; Oh, C.; Kim, M.S. The association between metabolic syndrome and iron status in pre-and postmenopausal women: KNHANES in 2012. Br. J. Nutr. 2021, 1-23. [CrossRef] [PubMed]

26. Duc, H.N.; Oh, H.; Yoon, I.M.; Kim, M.-S. Association between levels of thiamine intake, diabetes, cardiovascular diseases and depression in Korea: A national cross-sectional study. J. Nutr. Sci. 2021, 10, e31. [CrossRef]

27. Kwon, Y. Association of curry consumption with blood lipids and glucose levels. Nutr. Res. Pract. 2016, 10, 212-220. [CrossRef] [PubMed]

28. Paik, H.Y. Dietary Reference Intakes for Koreans (KDRIs). Asia Pac. J. Clin. Nutr. 2008, 17, 416-419.

29. Hosmer, D.W., Jr.; Lemeshow, S.; Sturdivant, R.X. Applied Logistic Regression; John Wiley \& Sons: New York, NY, USA, 2013; Volume 398.

30. Wang, M.; Liu, R.; Chen, W.; Peng, C.; Markert, B. Effects of urbanization on heavy metal accumulation in surface soils, Beijing. J. Environ. Sci. 2018, 64, 328-334. [CrossRef]

31. Czeisler, M.É.; Lane, R.I.; Petrosky, E.; Wiley, J.F.; Christensen, A.; Njai, R.; Weaver, M.D.; Robbins, R.; Facer-Childs, E.R.; Barger, L.K. Mental health, substance use, and suicidal ideation during the COVID-19 pandemic-United States, June 24-30, 2020. Morb. Mortal. Wkly. Rep. 2020, 69, 1049. [CrossRef]

32. Kelishadi, R.; Askarieh, A.; Motlagh, M.E.; Tajadini, M.; Heshmat, R.; Ardalan, G.; Fallahi, S.; Poursafa, P. Association of blood cadmium level with cardiometabolic risk factors and liver enzymes in a nationally representative sample of adolescents: The CASPIAN-III study. J. Environ. Public Health 2013, 2013. [CrossRef]

33. Gupta, V.K.; Singh, S.; Agrawal, A.; Siddiqi, N.J.; Sharma, B. Phytochemicals mediated remediation of neurotoxicity induced by heavy metals. Biochem. Res. Int. 2015, 2015, 534769. [CrossRef]

34. Jomova, K.; Valko, M. Advances in metal-induced oxidative stress and human disease. Toxicology 2011, 283, 65-87. [CrossRef]

35. Song, Y.; Yang, S.K.; Kim, J.; Lee, D.-C. Association between C-reactive protein and metabolic syndrome in Korean adults. Korean J. Fam. Med. 2019, 40, 116. [CrossRef] [PubMed]

36. Vinh quoc Luong, K.; Nguyen, L.T.H. The impact of thiamine treatment in the diabetes mellitus. J. Clin. Med. Res. $2012,4,153$.

37. Thornalley, P.; Babaei-Jadidi, R.; Al Ali, H.; Rabbani, N.; Antonysunil, A.; Larkin, J.; Ahmed, A.; Rayman, G.; Bodmer, C. High prevalence of low plasma thiamine concentration in diabetes linked to a marker of vascular disease. Diabetologia 2007, 50, 2164-2170. [CrossRef]

38. Thameem, F.; Wolford, J.K.; Bogardus, C.; Prochazka, M. Analysis of slc19a2, on 1q23. 3 encoding a thiamine transporter as a candidate gene for type 2 diabetes mellitus in pima indians. Mol. Genet. Metab. 2001, 72, 360-363. [CrossRef]

39. Kalis, M.; Kumar, R.; Janciauskiene, S.; Salehi, A.; Cilio, C.M. $\alpha$ 1-antitrypsin enhances insulin secretion and prevents cytokinemediated apoptosis in pancreatic $\beta$-cells. Islets 2010, 2, 185-189. [CrossRef] [PubMed]

40. Rodríguez, M.R. Importance of water-soluble vitamins as regulatory factors of genetic expression. Rev. Investig. Clin. Organo Hosp. Enferm. Nutr. 2002, 54, 77.

41. Yang, Z.; Ge, J.; Yin, W.; Shen, H.; Liu, H.; Guo, Y. The expression of p53, MDM2 and Ref1 gene in cultured retina neurons of SD rats treated with vitamin B1 and/or elevated pressure. Yan ke xue bao (2016) 2004, 20, 259-263.

42. Berrone, E.; Beltramo, E.; Solimine, C.; Ape, A.U.; Porta, M. Regulation of intracellular glucose and polyol pathway by thiamine and benfotiamine in vascular cells cultured in high glucose. J. Biol. Chem. 2006, 281, 9307-9313. [CrossRef]

43. Ayo, S.H.; Radnik, R.; Garoni, J.; Troyer, D.A.; Kreisberg, J.I. High glucose increases diacylglycerol mass and activates protein kinase C in mesangial cell cultures. Am. J. Physiol. Ren. Physiol. 1991, 261, F571-F577. [CrossRef]

44. Babaei-Jadidi, R.; Karachalias, N.; Ahmed, N.; Battah, S.; Thornalley, P.J. Prevention of incipient diabetic nephropathy by high-dose thiamine and benfotiamine. Diabetes 2003, 52, 2110-2120. [CrossRef]

45. Kohda, Y.; Shirakawa, H.; Yamane, K.; Otsuka, K.; Kono, T.; Terasaki, F.; Tanaka, T. Prevention of incipient diabetic cardiomyopathy by high-dose thiamine. J. Toxicol. Sci. 2008, 33, 459-472. [CrossRef]

46. González-Ortiz, M.; Martínez-Abundis, E.; Robles-Cervantes, J.A.; Ramírez-Ramírez, V.; Ramos-Zavala, M.G. Effect of thiamine administration on metabolic profile, cytokines and inflammatory markers in drug-naïve patients with type 2 diabetes. Eur. J. Nutr. 2011, 50, 145-149. [CrossRef] [PubMed] 
47. Al-Attas, O.; Al-Daghri, N.; Alokail, M.; Abd-Alrahman, S.; Vinodson, B.; Sabico, S. Metabolic benefits of six-month thiamine supplementation in patients with and without diabetes mellitus type 2. Clin. Med. Insights Endocrinol. Diabetes 2014, 7, CMED. S13573. [CrossRef]

48. La Selva, M.; Beltramo, E.; Pagnozzi, F.; Bena, E.; Molinatti, P.; Molinatti, G.M.; Porta, M. Thiamine corrects delayed replication and decreases production of lactate and advanced glycation end-products in bovine retinal and human umbilical vein endothelial cells cultured under high glucose conditions. Diabetologia 1996, 39, 1263-1268. [CrossRef] [PubMed]

49. Avena, R.; Arora, S.; Carmody, B.J.; Cosby, K.; Sidawy, A.N. Thiamine (vitamin B1) protects against glucose-and insulin-mediated proliferation of human infragenicular arterial smooth muscle cells. Ann. Vasc. Surg. 2000, 14, 37-43. [CrossRef] [PubMed]

50. Arora, S.; Lidor, A.; Abularrage, C.J.; Weiswasser, J.M.; Nylen, E.; Kellicut, D.; Sidawy, A.N. Thiamine (vitamin B1) improves endothelium-dependent vasodilatation in the presence of hyperglycemia. Ann. Vasc. Surg. 2006, 20, 653-658. [CrossRef] [PubMed]

51. Stirban, A.; Nandrean, S.; Kirana, S.; Götting, C.; Veresiu, I.A.; Tschoepe, D. Benfotiamine counteracts smoking-induced vascular dysfunction in healthy smokers. Int. J. Vasc. Med. 2012, 2012, 968761. [CrossRef]

52. Avignon, A.; Hokayem, M.; Bisbal, C.; Lambert, K. Dietary antioxidants: Do they have a role to play in the ongoing fight against abnormal glucose metabolism? Nutrition 2012, 28, 715-721. [CrossRef]

53. Palmieri, V.O.; Grattagliano, I.; Portincasa, P.; Palasciano, G. Systemic oxidative alterations are associated with visceral adiposity and liver steatosis in patients with metabolic syndrome. J. Nutr. 2006, 136, 3022-3026. [CrossRef]

54. Mazur-Bialy, A.I.; Pocheć, E. Riboflavin reduces pro-inflammatory activation of adipocyte-macrophage co-culture. Potential application of vitamin B2 enrichment for attenuation of insulin resistance and metabolic syndrome development. Molecules 2016, 21, 1724. [CrossRef]

55. Abdullah, K.; Alam, M.M.; Iqbal, Z.; Naseem, I. Therapeutic effect of vitamin B3 on hyperglycemia, oxidative stress and DNA damage in alloxan induced diabetic rat model. Biomed. Pharmacother. 2018, 105, 1223-1231. [CrossRef] [PubMed]

56. Mazur-Bialy, A.I.; Pocheć, E. Vitamin B2 deficiency enhances the pro-inflammatory activity of adipocyte, consequences for insulin resistance and metabolic syndrome development. Life Sci. 2017, 178, 9-16. [CrossRef] [PubMed]

57. Abdullah, K.; Qais, F.A.; Ahmad, I.; Naseem, I. Inhibitory effect of vitamin B3 against glycation and reactive oxygen species production in HSA: An in vitro approach. Arch. Biochem. Biophys. 2017, 627, 21-29. [CrossRef]

58. Adiels, M.; Chapman, M.J.; Robillard, P.; Krempf, M.; Laville, M.; Borén, J.; Group, N.S. Niacin action in the atherogenic mixed dyslipidemia of metabolic syndrome: Insights from metabolic biomarker profiling and network analysis. J. Clin. Lipidol. 2018, 12, 810-821. [CrossRef]

59. Khalil, A.; Gaudreau, P.; Cherki, M.; Wagner, R.; Tessier, D.M.; Fulop, T.; Shatenstein, B. Antioxidant-rich food intakes and their association with blood total antioxidant status and vitamin C and E levels in community-dwelling seniors from the Quebec longitudinal study NuAge. Exp. Gerontol. 2011, 46, 475-481. [CrossRef] [PubMed]

60. Srinivasan, M. Effect of curcumin on blood sugar as seen in a diabetic subject. Indian J. Med Sci. 1972, 26, 269.

61. Usharani, P.; Mateen, A.; Naidu, M.; Raju, Y.; Chandra, N. Effect of NCB-02, atorvastatin and placebo on endothelial function, oxidative stress and inflammatory markers in patients with type 2 diabetes mellitus. Drugs $R$ D 2008, 9, 243-250. [CrossRef]

62. Chuengsamarn, S.; Rattanamongkolgul, S.; Luechapudiporn, R.; Phisalaphong, C.; Jirawatnotai, S. Curcumin extract for prevention of type 2 diabetes. Diabetes Care 2012, 35, 2121-2127. [CrossRef]

63. Wickenberg, J.; Ingemansson, S.L.; Hlebowicz, J. Effects of Curcuma longa (turmeric) on postprandial plasma glucose and insulin in healthy subjects. Nutr. J. 2010, 9, 43. [CrossRef]

64. Alwi, I.; Santoso, T.; Suyono, S.; Sutrisna, B.; Suyatna, F.D.; Kresno, S.B.; Ernie, S. The effect of curcumin on lipid level in patients with acute coronary syndrome. Acta Med. Indones. 2008, 40, 201-210.

65. Lan, C.; Chen, X.; Zhang, Y.; Wang, W.; Wang, W.E.; Liu, Y.; Cai, Y.; Ren, H.; Zheng, S.; Zhou, L. Curcumin prevents strokes in stroke-prone spontaneously hypertensive rats by improving vascular endothelial function. BMC Cardiovasc. Disord. 2018, 18, 1-10. [CrossRef]

66. Liu, Z.; Ran, Y.; Huang, S.; Wen, S.; Zhang, W.; Liu, X.; Ji, Z.; Geng, X.; Ji, X.; Du, H. Curcumin protects against ischemic stroke by titrating microglia/macrophage polarization. Front. Aging Neurosci. 2017, 9, 233. [CrossRef] [PubMed]

67. Lim, S.; Min, S.H.; Lee, J.H.; Kim, L.K.; Lee, D.-H.; Lee, J.-E.; Kim, K.M.; Lee, S.; Park, K.-C.; Lee, Y.J. Components of metabolic syndrome in Korean adults: A hospital-based cohort at Seoul National University Bundang Hospital. J. Obes. Metab. Syndr. 2019, 28, 118. [CrossRef]

68. Huh, J.H.; Lee, J.H.; Moon, J.S.; Sung, K.C.; Kim, J.Y.; Kang, D.R. Metabolic syndrome severity score in Korean adults: Analysis of the 2010-2015 Korea National Health and Nutrition Examination Survey. J. Korean Med Sci. 2019, 34. [CrossRef] [PubMed]

69. Lee, J.-M.; Seok, K.-J.; Ryu, J.-Y.; Jung, W.-S.; Park, J.-B.; Shin, K.-H.; Jang, S.-J. Association between heavy metal exposure and prevalence of metabolic syndrome in adults of South Korea. Korean J. Fam. Pract. 2017, 7, 172-178. [CrossRef]

70. The Lancet. COVID-19: A new lens for non-communicable diseases. Lancet 2020, 396, 649. [CrossRef] 